\title{
WHOOPING CRANE RECORDS FOR MANITOBA, 1943-1979
}

PETER BOOTHROYD, Canadian Wildlife Service, 501 University Cresc., Winnipeg, Manitoba, R3T 2 N6.

On 5 June 1978, Ken Melnick, Manitoba Department of Natural Resources, and I took off in a Cessna 180 from the northern end of Fidler Lake to survey waterfowl breeding on the Churchill River in northern Manitoba. We went south, at an altitude of 30-60 metres and at a speed of 135-160 kph, along the eastern shoreline of the lake just south of where the Churchill River heads east. We observed four Sandhill Cranes standing in a deltalike area of mudflats where a stream entered the lake $\left(57^{\circ} 10^{\prime} \mathrm{N}, 96^{\circ} 54^{\prime} \mathrm{W}\right)$. A little further south, we caught sight of a Whooping Crane in flight approximately 15 metres above ground level: The crane passed directly beneath us so identification was easy. The long outstretched neck, the long legs trailing behind the bird, large white wings with black tips and red head patch were all clearly visible. The weather was overcast and viewing conditions were excellent with no glare or reflection from the sun to interfere with observations. We circled to make further observations of the bird and obtain photographs but were unable to find it again. We flew east to where the crane had come from in an attempt to locate other Whooping Cranes but found none.

As we continued our survey along the shoreline at the southern end of Fidler Lake, I was impressed by the similarity between some of the habitat located a short distance inland and habitat portrayed in photographs of Whooping Crane breeding grounds in
Wood Buffalo National Park, N.W.T. Numerous shallow ponds fringed with aquatic emergent vegetation interspersed by low ridges of black spruce, tamarack and willow occurred in the area. This type of habitat is not restricted to the Fidler Lake region but extends in a wide band from Hudson Bay across Manitoba, Saskatchewan and Alberta to the Northwest Territories.

It was exciting enough to witness an observation of a Whooping Crane in this part of Manitoba. However, the sighting was all the more interesting in the light of other reports of the species in northern Manitoba. ${ }^{1}$ I decided to attempt to locate other Whooping Crane records in the province, and am grateful to R. W. Nero of the Manitoba Department of Natural Resources and to H. W. R. Copland of the Manitoba Museum of Man and Nature for allowing me access to records in their files. Each record was examined and its authenticity determined on the basis of the amount and conformity of detail contained in the description of the observed bird(s) and presumed credibility of the observer. Those records considered to be authentic were divided into two categories, accepted and confirmed according to criteria used by W. J. D. Stephen, Canadian Wildlife Service, Saskatoon. ${ }^{2}$ Records in these categories are listed in Table 1. Locations of accepted records are plotted in Figure 1.

The major flight path for migrating 


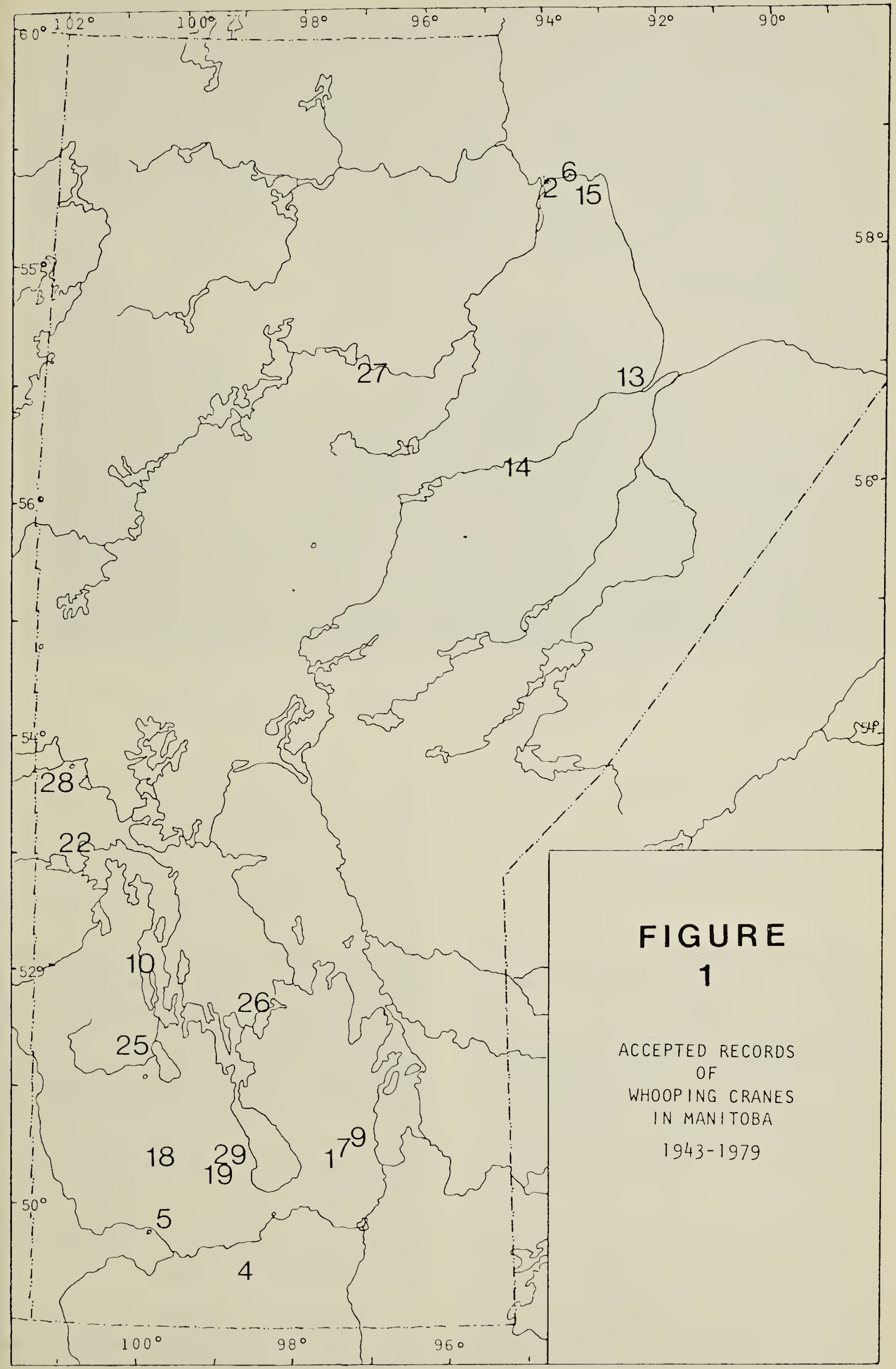

September, 1980. 38(3) 


\begin{tabular}{|c|c|c|c|c|c|c|c|c|c|c|}
\hline $\begin{array}{l}\text { Record } \\
\text { No. }\end{array}$ & Oate & Locat io & & Twp. & $\begin{array}{l}\text { Lega } \\
\text { Rge. }\end{array}$ & mer. & $\begin{array}{l}\text { No. of } \\
\text { Birds }\end{array}$ & $\begin{array}{l}\text { Move- } \\
\text { ment }\end{array}$ & Observer & $\begin{array}{l}\text { Type } \\
\text { Obser } \\
\text { vatior }\end{array}$ \\
\hline 1 & April 26, 1943 & East Shoal L. & $50^{\circ} 20^{\prime} \mathrm{N} 97^{\circ} 35^{\prime} \mathrm{W}$ & 17 & 23 & $w 1$ & 9 & N & F.C. Ward & $A^{1}$ \\
\hline 2 & oct. 1.1953 & Churchill & $58^{\circ} 45^{\prime} \mathrm{N} 94^{\circ} 35^{\prime} \mathrm{W}$ & not & appli & $a b l e$ & 3 & - & Mrs. E. Beckett & A \\
\hline 3 & April 28, 1955 & Russell & $50^{\circ} 55^{\prime} \mathrm{N} 10 P^{\circ} 19^{\prime} \mathrm{W}$ & 22 & 28 & WI & 4 & - & Mrs. R. Anderson & $u^{2}$ \\
\hline 4 & oct. $\quad 2,1955$ & Swan Lake & $49^{\circ} 25^{\prime} \mathrm{N} 98^{\circ} 50^{\prime} \mathrm{W}$ & not & appll & $a b l e$ & 2 & s & F.C. ward & A \\
\hline 5 & April 19. 1956 & Brandon & $49^{\circ} 50^{\prime} \mathrm{N} 100^{\circ} 00^{\prime} \mathrm{W}$ & 10 & 19 & $W 1$ & 2 & N & D.A. Ball & A \\
\hline 6 & sept. 6, 1964 & Churchill & $58^{\circ} 45^{\prime} \mathrm{N} 94^{\circ} 05^{\prime} \mathrm{W}$ & not & appli & $a b l e$ & 3 & - & 1.H. Smith & A \\
\hline 7 & April 25. 1971 & Teulon & $50^{\circ} 18^{\circ} \mathrm{N} 97^{\circ} 26^{\prime} \mathrm{W}$ & 15 & 1 & EI & 4 & - & R.C.M.P. & A \\
\hline 8 & April 29, 1973 & Ponemah & $50^{\circ} 28^{\prime} \mathrm{N} 96^{\circ} 57^{\prime} \mathrm{W}$ & 17 & 4 & El & 5 & $E$ & L. LaRue & $u$ \\
\hline 9 & May 17,1973 & Komarno & $50^{\circ} 34^{\prime} \mathrm{N} 97^{\circ} 24^{\prime} \mathrm{W}$ & 18 & 1 & El & 2 & - & $\begin{array}{l}\text { A. Rollianyk } \\
\text { Mrs. C. Roinanyk }\end{array}$ & A \\
\hline 10 & 8. 1974 & Camperville & $52^{\circ} 00^{\prime} \mathrm{N} 100^{\circ} 09^{\prime} \mathrm{W}$ & 35 & 20 & WI & 4 & SW & T.1. Smith & A \\
\hline 11 & 9. 1974 & Dauphin Beain & $51^{\circ} 08^{\prime} \mathrm{N} 99^{\circ} 50^{\prime} \mathrm{W}$ & 25 & 17 & WI & 2 & - & D. Juce & $u$ \\
\hline 12 & Late May, 1974 & Island Lake & $53^{\circ} 42^{\prime} \mathrm{N} 94^{\circ} 40^{\prime} \mathrm{W}$ & not & appli & $a b l e$ & 5 & NE & T.A. Hamilion & $u$ \\
\hline 13 & June 22,1974 & North Seal Ch. & $56^{\circ} 49^{\prime} \mathrm{N} 92^{\circ} 55^{\prime} \mathrm{W}$ & not & appli & able & 2 & - & $\begin{array}{l}\text { W. K. Harper } \\
\text { I.R. Buhuwsky }\end{array}$ & A \\
\hline 14 & Aug. 23,1974 & Gillan & $56^{\circ} 15^{\prime} \mathrm{N} 94^{\circ} 35^{\prime} \mathrm{W}$ & not & appli & able & 4 & - & D. Beckett & A \\
\hline 15 & April 27, 1975 & Churchill & $58^{\circ} 46^{\prime} \mathrm{N} 94^{\circ} 00^{\prime} \mathrm{W}$ & not & appli & $a b l e$ & 2 & E & $\begin{array}{l}\text { B. Erickson } \\
\text { Mrs. D. Erickson }\end{array}$ & A \\
\hline 16 & May 1.1975 & Red Deer Lahe & $52^{\circ} 55^{\prime} \mathrm{N} 10 P^{\circ} 27^{\prime} \mathrm{W}$ & 48 & 28 & WI & 2 & N & $\begin{array}{l}\text { B. Hather' } \\
\text { R: Hather }\end{array}$ & $u$ \\
\hline 17 & Late June. 1975 & Pinama & $50^{\circ} 09^{\prime} \mathrm{N} 95^{\circ} 55^{\prime} \mathrm{w}$ & 14 & 12 & EI & 4 & NE & w. Schwartz & $u$ \\
\hline 18 & April 6. 1976 & Stroal Lahe & $50^{\circ} 28^{\prime} \times 100^{\circ} 45^{\prime} \mathrm{W}$ & 17 & 24 & WI & 8 & $\mathrm{NW}$ & C.C. Findlay & A \\
\hline 19 & April 17. 1976 & Waldersee & $50^{\circ} 30^{\prime} \mathrm{N} 98^{\circ} 58^{\prime} \mathrm{W}$ & 18 & 11 & WI & 1 & - & E. Hoehn & A \\
\hline 20 & 3. 1977 & Pointe du buis & $50^{\circ} 13^{\prime} \mathrm{N} 95^{\circ} 34^{\prime} \mathrm{W}$ & 15 & 14 & EI & 7 & - & R. Knox & $u$ \\
\hline 21 & 8,1377 & Breezy Pount & $50^{\circ} 14^{\prime} \mathrm{N} 96^{\circ} 50^{\prime} \mathrm{W}$ & 14 & 5 & EI & 2 & $\mathrm{NW}$ & R. Allan & $u$ \\
\hline 22 & 16.1977 & Overflowing $R$. & $53^{\circ} 11 \cdot \mathrm{N} 10$ OPOS'W & 48 & 25 & $w 1$ & 1 & - & R.J. Robertson & A \\
\hline 23 & 18.1977 & Gildert Plains & $51^{\circ} 13^{\prime} \mathrm{N} 100^{\circ} 29^{\prime} \mathrm{W}$ & 25 & 22 & w1 & 4 & - & M. Stoughton & $u$ \\
\hline 24 & May 22,1977 & Gull Lahe & $50^{\circ} 24^{\prime} \mathrm{N} 96^{\circ} 31^{\prime} \mathrm{W}$ & 16 & 7 & EI & 3 & $\mathrm{NW}$ & A.R. Kear & $u$ \\
\hline 25 & April 11, 1978 & Ethelbert & $51^{\circ} 23^{\prime} \mathrm{N} 100^{\circ} 08^{\prime} \mathrm{W}$ & 26 & 18 & $w 1$ & 5 & - & M. Dohan & A \\
\hline 26 & April 16. 1978 & Gypsunville & $51^{\circ} 46^{\prime} \mathrm{N} 98^{\circ} 38^{\prime} \mathrm{W}$ & 32 & 8 & $W 1$ & 2 & - & C.J. Ruhr & A \\
\hline 27 & June $\quad 5,1978$ & Fidler Lake & $57^{\circ} 10^{\prime} \mathrm{N} 96^{\circ} 54^{\prime} \mathrm{W}$ & not & appli & able & 1 & $w$ & K. Melnick & A \\
\hline 28 & May 15, 1979 & Pasquia Lahe & $53^{\circ} 38^{\prime} \mathrm{N} 10 \mathrm{P} 24^{\prime} \mathrm{W}$ & 54 & 27 & $w 1$ & 1 & N & E.F. Whitney & A \\
\hline $2 \dot{y}$ & Sepr. 14, 1979 & Alitaranth & $50^{\circ} 30^{\prime} \mathrm{N} 98^{\circ} 44^{\circ} \mathrm{W}$ & 20 & 12 & $w 1$ & 3 & SE & C.W. Collins & $u$ \\
\hline
\end{tabular}

Accepted recoro

2 unconfirated record

Whooping Cranes has been stated as passing through Saskatchewan. ${ }^{3}$ However, sightings made in the prairie provinces in 1977 and 1978 indicate that this corridor extends to the east and west of Saskatchewan. ${ }^{2}$ Locations of sightings in southern and western Manitoba, illustrated in Figure 1 of this paper, support this observation. The sightings in northeastern Manitoba are particularly interesting since summer locations of all Whooping Cranes are not known. Most yearlings and other sexually immature Whooping Cranes are known to summer in the Northwest Territories portion of Wood Buffalo
National Park. ${ }^{4}$ However, to date, attempts have not been made to identify the summer locations of the entire Whooping Crane population (E. Kuyt, pers. comm.). The presence of habitat apparently suitable for Whooping Cranes in numerous uninhabited regions of northern Manitoba, and the dates of some of the Manitoba sightings (Table 1), leads to the speculation that some cranes may spend a portion of the summer in Manitoba in some years.

If Whooping Cranes are indeed summering in Manitoba, acceptance of sightings will be enhanced if obser- 


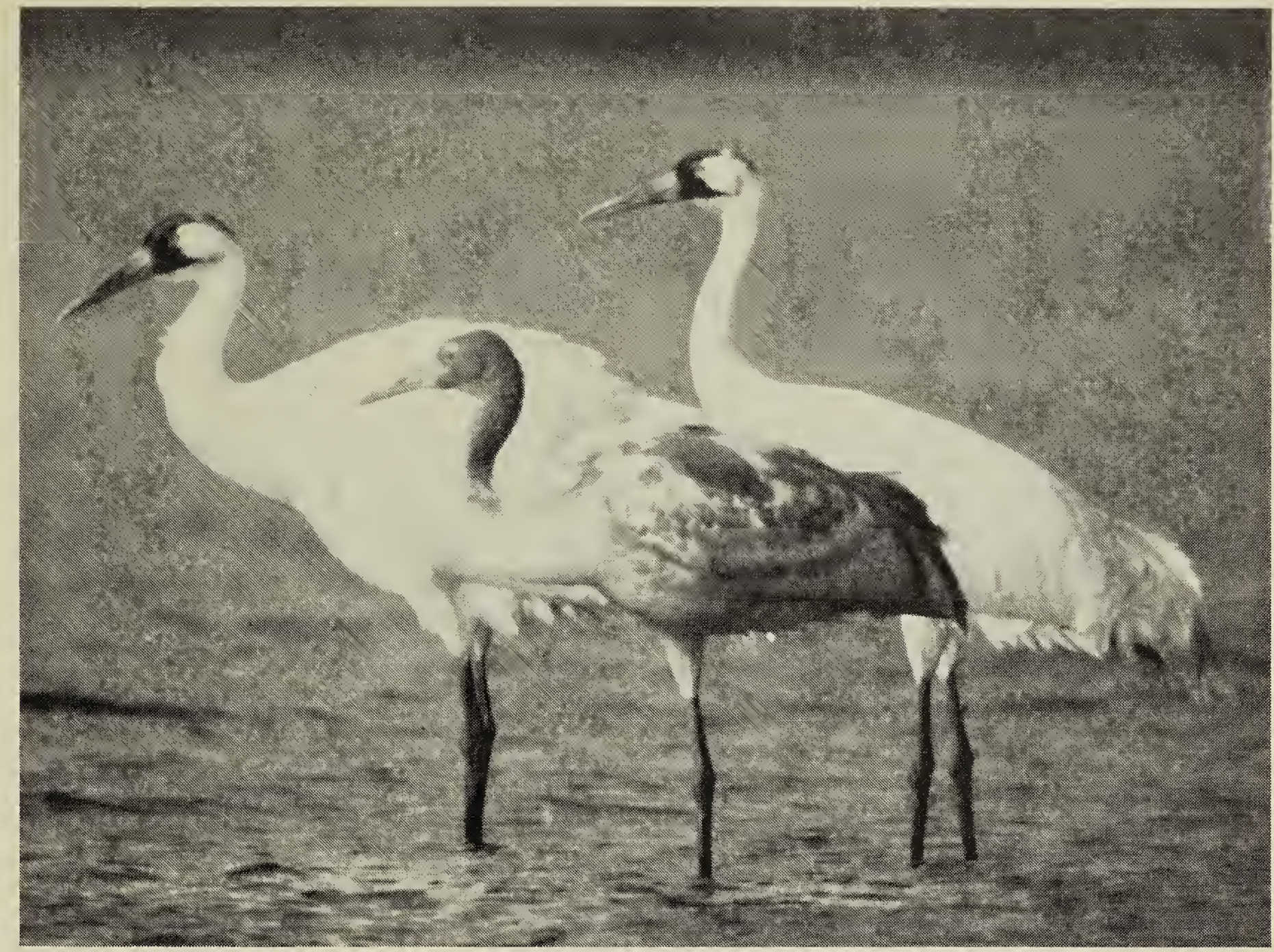

Adult Whooping Cranes with young, Avonlea, Saskatchewan.

Lorne Scott

vations made are complete. Man's ability to preserve this endangered species and ensure its future existence depends, in part, on the extent of our knowledge of its distribution, movements and habits. The opportunity to contribute to this knowledge is available to us all. I would urge anyone who observes a Whooping Crane to report the sighting to: Mr. E. Kuyt - Canadian Wildlife Service, 100 , 9942-108th Street, Edmonton, Alberta, T5K 2J5, (403) 420-2516.

'DIDIUK, A. 1975. Whooping cranes in
Manitoba? Manitoba Nature 16(3):3439.

${ }^{3}$ STEPHEN, W. J. D. 1979. Whooping crane sightings prairie provinces 1977 and 1978. Blue Jay $37(3): 163-168$.

${ }^{3}$ BARD, F. G. 1959. Annual report of whooping cranes in Saskatchewan, 1958. Blue Jay 17(1):9-13.

${ }^{4} \mathrm{KUYT}$, E. 1979. Banding of juvenile whooping cranes and discovery of the summer habitat used by nonbreeders. In J. C. Lewis, (Ed.), Proceedings of the 1978 Crane Workshop. Colorado State U., Ft. Collins. 259 pp. 